\title{
El carbono orgánico del suelo y su papel frente al cambio climático
}

\author{
The soil organic carbon and its role on climate change
}

\section{Hernán Burbano Orjuela ${ }^{1}$}

1 Ingeniero Agrónomo, M. Sc., Doctor Honoris Causa. Profesor titular y distinguido de la Universidad de Nariño (Jubilado), Pasto, Colombia, hernan.burbano@googlemail.com

Citar: Burbano-Orjuela, H. (2018). El carbono orgánico del suelo y su papel frente al cambio climático. Rev. Cienc. Agr. 35(1): 82-96. doi: http://dx.doi.org/10.22267/rcia.183501.85.

Recibido: Febrero 2 de 2018. Aceptado: Abril 10 de 2018.

\section{RESUMEN}

El cambio climático es un problema ambiental al que debe hacer frente la sociedad mundial, sin embargo, hay alternativas para mitigar dicho fenómeno y en ese contexto, el suelo puede jugar un papel importante. Por estas razones, en este artículo se hace consideraciones sobre la potencialidad del suelo para almacenar carbono, siempre en el marco de procesos mayores como el ciclo del carbono y el propio cambio climático. Por último, se destaca una iniciativa mundial para manejar el suelo en función de la problemática mencionada.

Palabras clave: Gases de efecto invernadero, clima, ecosistemas terrestres, materia orgánica.

\begin{abstract}
Climate change, one of the most important global challenges, is an unavoidable problem for society. Soil, among other alternatives, can play a role mitigating the detrimental effects of CO2-driven climate change. In this article, I discuss soil's potential to store carbon in the context of the carbon cycle and its possible role on the modulation of climate change. Finally, I explain a global initiative of soil management as an approach to mitigate climate change.
\end{abstract}

Key words: Greenhouse gases, climate, terrestrial ecosystems, organic matter. 


\section{INTRODUCCIÓN}

Se suele considerar que el cambio climático (CC) solo tiene que ver con lo que ocurre en la atmósfera, no obstante, por acción de la fotosíntesis que utiliza el carbono atmosférico, una proporción de dicho carbono llega al suelo y se puede estabilizar por periodos tan amplios que pueden ser miles de años. A la luz de esta consideración, los suelos en mayor o menor proporción pueden coadyuvar a los esfuerzos tendientes a disminuir el cambio climático (Agencia Europea del Medio Ambiente, 2015). Lo anterior, es posible porque el suelo además de prestar otros servicios ecosistémicos claves para todas las expresiones de vida en el planeta, desempeña un rol estratégico e insustituible en el ciclo del carbono en la Tierra. (Dimas y Gnacadja, 2009).

Resulta entonces, que en las condiciones a las que se está aludiendo, el suelo es un componente primordial del sistema climático del planeta, porque cuantitativamente es el segundo sumidero de carbono en la naturaleza, solo precedido por los océanos (Agencia Europea del Medio Ambiente, 2015). Lo anterior, se soporta en que el suelo juega activamente en la dinámica de generación y fijación 0 captura de los gases de efecto invernadero (GEI), cuyas concentraciones crecientes modifican los regímenes de temperatura y precipitación pluvial en lo regional, propiciando situaciones extremas de sequías e inundaciones que también alteran los procesos que regulan los suelos con respecto a los GEI y revierten adversamente sobre el propio suelo (Caviglia et al., 2016).

Es evidente que el suelo es un gran reservorio de carbono en la naturaleza (Dimas y Gnacadja, 2009). Ahora, el contenido de carbono en el suelo obedece a sus características y al equilibrio entre las tasas de entrada de carbono orgánico de origen vegetal y animal, y las de salida, resultantes del metabolismo microbiano que genera $\mathrm{CO}_{2}$. Como las tipologías de los suelos son diversas, hay que tomarlas en cuenta en los balances que determinan las cantidades de carbono orgánico en el suelo (COS), sumando a ello la influencia del clima y las prácticas de manejo adoptadas (Docampo, 2010).

El manejo del suelo puede direccionar su rol en las circunstancias del cambio climático, al afectar sus contenidos de carbono y llevar, aunque en principio resulte paradójico, a que el suelo en lugar de sumidero de carbono pase a ser un emisor importante de GEI. Por eso, en el mundo de hoy se busca que unas apropiadas prácticas agrícolas y silvícolas recuperen y mantengan la potencialidad de los suelos e influyan al tiempo en la cantidad de carbono que estos puedan almacenar (Agencia Europea del Medio Ambiente, 2015). En la misma dirección se propone una gestión sostenible de la tierra (GST) que obre en dos sentidos. La reconstrucción de las reservas de carbono en el suelo lo mismo que de la cobertura vegetal, y la disminución del $\mathrm{CO}_{2}$ atmosférico y la desaceleración del calentamiento global (Gabathuler et al., 2009).

Con estos referentes iniciales, se procede a realizar un análisis de cómo el suelo en su condición de un gran almacén de carbono en la naturaleza, puede, en el ámbito del cambio climático, contribuir a la disminución de los efectos negativos de este fenómeno global. Como en la naturaleza todo tiene que ver con todo, este ensayo toma en consideración el cambio climático y el ciclo del carbono subrayando los puntos de encuentro con el suelo, finalmente, se muestra una estrategia global en la cual se destaca la importancia del almacenamiento del carbono en el suelo.

\section{El cambio climático}

El clima ha estado ligado al discurrir del género humano, como que el despegue de la agricultura en el neolítico y el paso de los hombres de nómadas a sedentarios, fue la resultante de un clima más benigno ligado justamente al neolítico después de 
la última glaciación, aunque de ahí en adelante el éxito y el fracaso de las civilizaciones tuvo alianzas o antagonismos con el clima. La sinergia entre temperaturas más altas y precipitaciones adecuadas hicieron posible la domesticación de especies vegetales -fuentes de alimento- en diferentes partes del mundo, originando lo que algunos califican de primera revolución verde (Pardos, 2010).

La historia da cuenta de las variaciones del clima que ocurrían en periodos cortos medidos en años o tan amplios que cubrían milenios. Hoy existen evidencias científicas que registran una alteración climática mundial que se observa en los incrementos en el promedio global de la temperatura del aire y de los mares, en el deshielo creciente de glaciares y en el consiguiente ascenso del nivel medio del mar. Este conjunto de fenómenos y circunstancias se denomina cambio climático (CC) que, a juicio del Panel Intergubernamental de Expertos sobre el Cambio Climático (IPCC por sus siglas en inglés), tendría como causa la fuerza dominante de las acciones de origen humano, al punto que hoy se afirma que se vive una nueva época geológica denominada antropoceno (PNUMA-ORPALC / FSUNEP Centre, 2014; Equihua et al., 2016)

El cambio climático global es uno de los principales problemas ambientales que enfrenta la sociedad mundial y que trae consigo el incremento de la temperatura media planetaria, como consecuencia del aumento -de origen antrópico- de los GEI, sobre todo dióxido de carbono $\mathrm{CO}_{2}$, metano $\mathrm{CH}_{4} \mathrm{y}$ óxido de nitrógeno $\mathrm{N}_{2} \mathrm{O}$, en cuya dinámica el suelo como almacén de carbono orgánico ejerce una función clave. El efecto señalado irá creciendo en las próximas décadas y de ahí la preocupación mundial por este fenómeno (Vela et al., 2012; Pla, 2010; Orellana et al., 2012; Urquiaga et al., 2005).

Si bien, en el fenómeno del cambio climático se atribuye la mayor responsabilidad a la combustión de carburantes fósiles, se olvida al ciclo del ácido tricarboxílico (CTC) que genera tres moléculas de $\mathrm{CO}_{2}$ por vuelta. Por tanto, eucariotes y procariotes adicionan una buena cantidad de $\mathrm{CO}_{2}$, estimándose que una persona adulta genera un kilo de $\mathrm{CO}_{2}$ al día y que así mismo son grandes las cantidades que desprenden los microorganismos en los suelos agrícolas (Blasco y Burbano, 2015).

Información de los últimos 1000 años pone en evidencia que la concentración del $\mathrm{CO}_{2}$ pasó de $280 \mathrm{a}$ más de $360 \mathrm{mg} \mathrm{kg}^{-1}$ en 200 años y que más del $50 \%$ de este cambio se produjo después de 1950, a una tasa de $0,8 \mathrm{mg} \mathrm{kg}^{-1}$ por año (Sandoval et al., 2003). También se considera que la incorporación de $\mathrm{CO}_{2}$ a la atmósfera durante los últimos 20-25 años tuvo su origen en un $75 \%$ en la quema de combustibles fósiles y el $25 \%$ restante, sobre todo, como resultado de los cambios en el uso del suelo y muy particularmente de la deforestación. En estas circunstancias, se vaticina que la temperatura media superficial del planeta se incremente entre 1,4 y $5,8^{\circ} \mathrm{C}$ para el periodo 1990 2100 (Vela et al., 2012; Lal, 2003).

Los cambios en los patrones regionales de temperatura, precipitación pluvial y frecuencia de eventos extremos como sequías, inundaciones, olas de calor, heladas se deben a los cambios en el balance de energía del sistema climático. El calentamiento global se deriva de concentraciones incrementales de GEI en la atmósfera, que obran reteniendo el calor que se irradia desde la superficie del planeta, señalando que la atmósfera es el menor y el más dinámico de los reservorios del ciclo del carbono (Caviglia et al., 2016; Rügnitz, 2009).

Aunque dentro del grupo de estos gases el $\mathrm{CO}_{2}$ es el primero por volumen de emisiones, los otros poseen un mayor potencial de retención de calor. Así, el potencial de calentamiento global del $\mathrm{CH}_{4} \mathrm{y}$ del $\mathrm{N}_{2} \mathrm{O}$ es 21 y 310 veces más alto que el correspondiente al dióxido de carbono. El origen de las emisiones de $\mathrm{CH}_{4}$ radica en la fermentación digestiva de rumiantes y en la descomposición anaeróbica 
del COS en suelos inundados plantados con arroz (Caviglia et al., 2016). Por su parte, los procesos de desnitrificación en el suelo explican las emisiones de $\mathrm{N}_{2} \mathrm{O}$ a la atmósfera (Verhulst et al., 2015). El incremento de los GEI y el calentamiento global inciden negativamente en muchas actividades de interés socioeconómico, aunque un efecto que destaca por las connotaciones sobre la supervivencia de las personas, es que se está comprometiendo la seguridad alimentaria por merma en los rendimientos de los cultivos, con efectos más dramáticos en países en vías de desarrollo (Altieri y Nicholls, 2013).

A pesar de las dificultades propias del CC se vienen identificando opciones para bajar los niveles de los GEI. Entre estas se mencionan dos: Primera, la disminución de las emisiones antropogénicas. Segunda, la creación y mejoría de los sumideros de carbono en la biósfera. En favor de esta última, estarían la conservación de los bosques y el establecimiento de sistemas agroforestales (SAF) que, en conjunto, remueven carbono atmosférico, lo fijan y lo almacenan por un buen periodo de tiempo. Al proceder así, finalmente, se contribuye a un almacenamiento mucho más prolongado del carbono, que es el que se fija o incorpora a la materia orgánica del suelo (MOS) que hace que el suelo sea el mayor sumidero de carbono en la naturaleza (Gallardo, 2002; Vela et al. 2012). Los GEI funcionan como una capa que no posibilita el paso de radiaciones que deberían ser reflejadas fuera del planeta. No obstante, el efecto invernadero es un fenómeno natural, clave para que se dé la vida en la Tierra, ya que impide que el planeta se enfríe en demasía durante la noche, garantizando la vida de muchos organismos incluida la de la especie humana. El problema del efecto invernadero radica en que su acción se ha exacerbado por la desmesurada producción de GEI por interés exclusivo de los seres humanos (PNUMA-ORPALC / FS-UNEP Centre, 2014).

Resulta claro que las investigaciones sobre el efecto invernadero y calentamiento de la Tierra han ubicado en la palestra al dióxido de carbono, con abundante información sobre su acumulación atmosférica. Aunque no hay que olvidar que este gas, al igual que el agua, absorbe las radiaciones infrarrojas, que no permiten que se escape el calor, produciéndose de esta forma el denominado efecto invernadero. También, al igual que el agua el dióxido de carbono es necesario para mantener la vida en la Tierra (Blasco y Burbano, 2015).

Por el impacto que tiene sobre la sociedad mundial el CC hace parte de la Agenda 2030 para el Desarrollo Sostenible, orientada a eliminar la pobreza y el hambre, proteger el planeta y garantizar la prosperidad de toda la población. Por eso la FAO considera que el CC y la reducción del riesgo de desastres constituyen el epicentro de su actual "marco estratégico". Lo anterior, demanda un cambio de enfoque con sistemas agroalimentarios que privilegien la resiliencia, la productividad y la sostenibilidad (FA0, 2015).

\section{El ciclo del carbono}

El carbono es el elemento químico clave en los compuestos de naturaleza orgánica, elemento que circula entre los océanos, la atmósfera, el suelo y el subsuelo, instancias que conforman los depósitos, reservorios o almacenes de carbono. El paso entre los depósitos ocurre mediante procesos de orden químico, físico y biológico. El intercambio de carbono entre el reservorio terrestre y el atmosférico es el resultado de procesos naturales como la fotosíntesis y la respiración, así como de la emisión de gases de origen antrópico (Rügnitz et al., 2009)

El ciclo del carbono se inicia con la fijación del dióxido de carbono atmosférico mediante la fotosíntesis que realizan las plantas y algunos microorganismos. En la fotosíntesis, el dióxido de carbono y el agua reaccionan para formar carbohidratos y a la vez, liberar oxígeno, que va a la atmósfera. Parte de los carbohidratos se consumen directamente 
para suministrar energía a la planta y el dióxido de carbono que así se forma, se libera a través de sus hojas o de sus raíces. Otra parte la consumen los animales, que también liberan dióxido de carbono en sus procesos metabólicos. Las plantas y los animales muertos, en últimas, son descompuestos por los microorganismos del suelo y por ello el carbono de sus tejidos se oxida, forma dióxido de carbono y retorna a la atmósfera (Orellana et al. 2012). En correlación con lo anterior hay que mencionar que en el suelo ocurre respiración, que consiste en la producción de $\mathrm{CO}_{2}$ resultante de dos procesos: la ruptura u oxidación de la MOS realizada por los microorganismos del suelo, y la respiración de las células de las raíces de las plantas. La tasa de producción de $\mathrm{CO}_{2}$ es importante porque es indicativa de la tasa de descomposición de la MOS y en consecuencia, de la cantidad de carbono que se pierde del sistema suelo. Las medidas de la respiración del suelo, en consecuencia, ayudan a determinar la contribución del suelo al balance del $\mathrm{CO}_{2}$ en la atmósfera (Lessard et al., 2006).

Se considera que el ciclo global del carbono consiste de dos ciclos. Uno bioquímico a corto plazo y otro geoquímico a largo plazo. Las mediciones muestran que, anualmente, las actividades antropogénicas afectan los dos ciclos con la emisión de 8,6Pg de carbono, siendo que el petagramo es igual a $10^{15}$ gramos o 1 billón de kilogramos. Se estima que del carbono emitido, 3.3Pg los absorbe la atmósfera y 2.2Pg los absorben los océanos. De estas cifras se colige que en los últimos 150 años hubo un incremento en las emisiones de $\mathrm{CO}_{2}$ a la atmósfera de un $31 \%$. Vale señalar el rol que cumple el suelo en este contexto, a partir de dos reservas, una, la de carbono orgánico del suelo (COS) y, otra, la de carbono inorgánico del suelo (CIS) (Verhulst et al., 2015).

Globalmente, el equilibrio del carbono en la Tierra está en función de tres reservorios, los océanos con una cantidad estimada de carbono de $38.000 \mathrm{Pg}$, la atmósfera con $750 \mathrm{Pg}$, y el sistema terrestre con
550Pg como biomasa-vegetación y 1.550Pg como COS. Los tres reservorios se hallan en un equilibrio dinámico, con interacciones entre unos y otros. Surge un cuarto reservorio, el geológico, que se estima tiene $65,5 \times 10^{6} \mathrm{Pg} \mathrm{C}$ y sólo una pequeña porción de éste que se podría aproximar a 4000Pg correspondería a combustible fósil. En el ecosistema terrestre, el mayor componente es el COS con $1.550 \mathrm{Pg} \mathrm{C}$, seguido por el CIS con 750-950Pg C. Se advierte que en las estimaciones globales del contenido de carbono en el suelo hay dificultades derivadas de alta variabilidad espacial del contenido de carbono en los suelos; una estimación relativa de las áreas ocupadas por distintos tipos de suelos; insuficientes datos de densidad aparente, necesaria para cuantificar la composición volumétrica y los complejos efectos de la vegetación y de los cambios en el uso del suelo (Sandoval et al., 2003; Martínez et al., 2008).

Es interesante saber que en el intercambio de $\mathrm{CO}_{2}$ entre la vegetación y la atmósfera ocurren cambios en los balances netos entre el secuestro -mejor llamado almacenamiento o fijación- y la liberación en diferentes periodos de tiempo, así: minuto a minuto, como cuando las nubes interceptan la luz solar; patrón día-noche, a lo largo de un ciclo estacional de predominancia del crecimiento y la descomposición; las etapas del ciclo de vida de la vegetación o del sistema de uso de la tierra (Kurniatun et al., 2011).

En la biosfera se debe diferenciar entre el carbono almacenado en el ecosistema -árboles, vegetación complementaria, suelo y productos obtenidosexpresado en toneladas o gigatoneladas por hectárea y el flujo de carbono, que es la corriente de carbono entre las existencias de carbono -contenido- en el ecosistema y la atmósfera. También, la expresión sumidero de carbono alude a la existencia de un flujo neto de carbono desde la atmósfera al sistema, en tanto que la expresión fuente de carbono se refiere a un flujo en sentido 
inverso, es decir, del sistema a la atmósfera. Se subraya que su diferencia algebraica se refleja en el balance de carbono (Pardos, 2010).

También es de interés considerar que, en una escala de tiempo estacional, la reserva de carbono en los ecosistemas y en los agroecosistemas se puede entender por los cambios en la biomasa vegetal 0 "variable rápida" aunque después de algunos años la reserva de carbono en el suelo o "variable lenta" resulta ser un indicador más importante acerca del funcionamiento del sistema, ligado a su capacidad de adaptación y resiliencia frente a efectos perturbadores y en consecuencia, a su capacidad a largo plazo para abastecer con bienes y servicios al ecosistema (UNCCD, 2015).

En el carbono puede circular por diferentes vías. En la mayoría de los años, el efecto anual neto de la fotosíntesis, la respiración y la descomposición da lugar a un incremento más bien pequeño del carbono que puede almacenarse. No obstante, las ganancias acumuladas en ciertas ocasiones se pierden, bien cuando hay años de inundaciones o ya cuando la materia orgánica se consume por acción del fuego. El carbono también puede ir a otros lugares. La madera, resinas, semillas, tubérculos, todos productos orgánicos, se mueven fuera de la zona donde se producen y se integran a los flujos de comercio, concentrándose principalmente en los sistemas urbanos y en sus vertederos de residuos. Unas pequeñas proporciones de las reservas de carbono alcanzan a filtrarse a través de la tierra e integrarse a sumideros de largo plazo como los cuerpos de agua dulce o el mar, también pueden aportar a la formación de turba (Kurniatun et al., 2011).

No es lo mismo verificar el cambio final en el total de carbono global, que contabilizar de manera sistemática todas las entradas y salidas de carbono, lo cual es un proceso más complejo. Al contabilizar las reservas de carbono de esta forma, se ha podido determinar que entre otros, el uso de la tierra, el cambio de uso de ésta y la silvicultura responden por el 15-20\% de las emisiones totales de GEI. También se sabe que en la zona templada ocurre secuestro neto de carbono, que los trópicos son responsables de las grandes emisiones netas, y que las zonas de turberas tropicales son fuentes pequeñas aunque con altos valores de emisión (Kurniatun et al., 2011).

El carbono del suelo está muy relacionado con el proceso de descomposición de la biomasa por la acción microbiana. Una parte del carbono del suelo vuelve a la atmósfera mediante la mineralización del carbono orgánico. Otra parte es conducida por las corrientes de los ríos hasta llegar al mar, donde se deposita en forma de carbonatos, proceso que es incentivado por la acción humana. El intercambio de carbono entre el reservorio marino y el atmosférico se da por procesos químicos que determinan "un equilibrio entre las camadas superficiales de los océanos y las concentraciones en el aire sobre la superficie". Ahora, la cantidad de $\mathrm{CO}_{2}$ que el mar absorbe, está en función de su temperatura y de la concentración de partida, por lo que temperaturas más altas del agua pueden generar la emisión de $\mathrm{CO}_{2}$ (Rügnitz et al., 2009).

La investigación y divulgación acerca del ciclo del carbono, en los últimos tiempos, se ha venido insertando en el ámbito del deterioro ambiental, donde el suelo resulta clave por su papel dual en el secuestro de carbono a corto y largo plazo y en la emisión en forma de $\mathrm{CO}_{2}$ a la atmósfera. También se valora que el COS determina la calidad del suelo en la medida en que participa en procesos bioquímicos y físicos que posibilitan la presencia de biomasa aérea y subterránea, que también actúa como reservorio de carbono en los ecosistemas terrestres (Fuentes et al., 2012). 


\section{El carbono orgánico del suelo y su relación con el cambio climático}

De acuerdo con Blasco y Burbano (2015), la materia orgánica del suelo es la fracción de la biosfera incorporada a la pedosfera. En los componentes se halla toda la materia muerta de origen eucariote y procariote, y sus productos metabólicos orgánicos. Generalmente, los suelos poseen cerca de un $5 \%$ de materia orgánica, en forma coloidal y en partículas. El suelo recibe compuestos orgánicos monoméricos y poliméricos de alto peso molecular -en mayor proporción-. Todos estos compuestos sufren transformación catabólica y finalmente dan los elementos precursores y junto con el carbono también están el hidrógeno, el oxígeno y el nitrógeno, a los cuales se suman otros elementos minerales acomplejados en los compuestos catabolizados. Cuando el proceso es natural o el suelo es objeto de manejo sostenible, como en todo metabolismo, la acción catabólica se compensa con la anabólica.

Cuando se hace alusión a la presencia de carbono en el suelo es para referirse al contenido de materia orgánica que es el propio carbono orgánico, sin que del mismo haga parte el carbono de la hojarasca o mantillo que se localizan sobre el suelo. El COS conforma cerca de los dos tercios del C fijado en los ecosistemas terrestres con un valor de $1.500 \mathrm{Pg} \mathrm{C}$. Por lo expresado, el suelo representa un gran almacén de carbono en la naturaleza (Pardos, 2010; Caviglia et al., 2016; PNUMA-ORPALC / FS-UNEP Centre, 2014).

El carbono del suelo se localiza en cuatro reservorios, dos de ellos muy grandes uno inorgánico y otro orgánico, y dos con menor participación, el atmosférico o sea el ubicado en el aire del suelo y aquel que está en solución (Blasco y Burbano, 2015). Además, el principal tipo de carbono orgánico en el suelo se halla en diferentes etapas de humificación, con plazos de recambio que alcanzan cientos o incluso miles de años. En las turberas, estos plazos pueden ser hasta de miles de años (Kurniatun et al., 2011). La dinámica de este carbono en el suelo depende de la actividad biológica, como que la mayor parte de los microorganismos de este medio son heterotróficos y por consiguiente demandan compuestos orgánicos preformados; a lo anterior se suma la estabilización de diferente grado que sufren los compuestos orgánicos por la interacción con la fracción mineral del suelo, especialmente, con las arcillas (Labrador, 2012).

Por sus variados orígenes el COS o la MOS no es un material homogéneo, por eso, se categoriza en dos fracciones. Una, la fracción lábil que es más susceptible a su descomposición o mineralización. Otra, la fracción recalcitrante y por lo mismo estable. En la primera se hallan materiales de escasa permanencia en el suelo como los residuos de plantas y microorganismos en diferentes estadios de descomposición. En la segunda, hay participación mayoritaria de sustancias húmicas de lenta descomposición, como resultado de su alto peso molecular, de sus estructuras complejas, irregulares y aromáticas y de su interacción con los constituyentes minerales del suelo. Por lo señalado, el COS lábil es más activo y de una respuesta más rápida a la gestión y al manejo que el COS estable. Conocer estas fracciones del COS es indispensable para entender y modelar su dinámica y para prever sus respuestas en el contexto del cambio climático global (Armas et al., 2013).

La presencia del carbono en el suelo se puede considerar respecto al carbono orgánico del suelo (COS) o de la materia orgánica del suelo (MOS), advirtiendo que el carbono tiene funciones nutricionales particulares y que el catabolismo de los microorganismos se realiza en los compuestos orgánicos, toda vez que la MOS es el consolidado de la materia orgánica muerta y la biomasa. La cantidad total de la MOS está en función del balance entre la productividad de la biomasa y la velocidad 
catabólica de la misma. Cuando el catabolismo es menor que la entrada de biomasa, la materia orgánica se acumula, como sucede en las turbas o en muchos suelos de regiones frías y húmedas. Si la tasa de descomposición supera a la adición de biomasa, la MOS disminuye, como ocurre en muchas áreas bajo cultivo, aunque también en esta disminución obran otros fenómenos denominados de exportación física, como la erosión y la percolación (Blasco y Burbano, 2015; UNCCD, 2015).

En general, los contenidos del COS tienden a disminuir con la profundidad. A la vez, los cambios más fuertes en la reserva de carbono en el suelo obedecen a las modificaciones en la cobertura terrestre y tiene lugar en los primeros $20-30 \mathrm{~cm}$, aunque en la práctica solo se suelen medir cambios en los primeros $5 \mathrm{~cm}$ superficiales (Kurniatun et al., 2011). Los cambios de uso del suelo como los que suceden cuando se deforestan los terrenos para hacer agricultura, no solo derivan en pérdidas de carbono del suelo sino que generan emisiones de GEI que contribuyen al cambio climático. Estos cambios de uso, se sabe, son responsables del $24 \%$ de las emisiones globales de dióxido de carbono (PNUMA-ORPALC / FS-UNEP Centre, 2014). En consecuencia, acciones inversas como la reforestación para suelos degradados es una alternativa importante de secuestro de carbono al largo plazo, tanto en la biomasa como en el suelo (Zambrano et al., 2004).

No obstante lo acabado de señalar, en el suelo ocurren procesos que hacen que el carbono pueda ser estabilizado de varias formas. Físicamente, cuando se forman agregados estables que lo protegen de la descomposición; químicamente, cuando el carbono se asocia con partículas minerales; bioquímicamente, cuando se forman compuestos recalcitrantes muy resistentes a la degradación microbiana. Ahora, como en la naturaleza los procesos son de ida y vuelta, el suelo puede funcionar como sumi- dero o como fuente de $\mathrm{CO}_{2}$ atmosférico, en función de las tasas de formación y descomposición del carbono orgánico del suelo (Caviglia et al., 2016; Martínez et al., 2008).

Si la concentración de dióxido de carbono en la atmósfera sigue incrementándose, esta situación puede propiciar que los microorganismos edáficos descompongan con mayor rapidez la MOS, pudiendo congruentemente, liberar más dióxido de carbono. No se sabe cuál puede ser el efecto total, porque según las regiones del mundo hay diferentes niveles de absorción y emisión de GEI. Aunque, de todas maneras, existe el riesgo de que el calentamiento de la atmósfera lleve a que el suelo libere mayor cantidad de GEI, dando lugar a lo que se viene denominando un "círculo vicioso" que impulse aún más el cambio climático (Agencia Europea del Medio Ambiente, 2015).

El incremento de la temperatura también tiene un efecto evidente en la captura de carbono. Dicho aumento podría inducir una mayor tasa de desmantelamiento o de mineralización de la MOS por los microorganismos, lo mismo que una mayor tasa de respiración de las raíces. Esta realidad podría ser más contundente en las regiones o países más fríos en donde cabría esperar un aumento en las emisiones de dióxido de carbono. Aunque se opina también, que en la mayor parte del planeta sería de prever una mayor captura de carbono (FAO, 2002).

Con el cambio climático y el carbono pueden darse dos situaciones. Si el carbono integra los GEI, este elemento hace parte del problema. Surge como solución cuando está en su forma orgánica en el suelo. Cuantitativamente el carbono del primer metro de suelo es el doble y el triple, que el de la atmósfera y el de la vegetación total. Por tanto, subir el carbono del suelo es una reserva clave que puede contrarrestar las emisiones de GEI. Además, el carbono contribuye a la fertilidad y así a la génesis de los ecosistemas naturales y agrícolas 
que, en encadenamiento, suplen las necesidades alimenticias de la población mundial, abastecen los recursos naturales y mantienen la biodiversidad. Por ello, el mundo natural y social se torna resiliente cuando dispone de suelos productivos. El carbono, entonces, es poli-funcional y es "una relación de uno a muchos, la misma molécula de carbono que se mantiene o añade al suelo la que trae todos estos beneficios en simultánea". Por eso, se pide adoptar una agricultura de conservación con una gestión sostenible de la tierra (GST) para aumentar los contenidos de carbono en el suelo y reducir la emisión de $\mathrm{CO}_{2}$, para salir al paso de los grandes retos ambientales: cambio climático, degradación de tierras y pérdida de diversidad biológica. Lo anterior, al amparo de políticas mundiales que optimicen el COS, para el bienestar humano y planetario (UNCCD, 2015; Cotler et al., 2016).

Desde otra perspectiva, se opina que la captura de carbono es un compromiso para ofrecer alternativas favorables y nuevos beneficios a las comunidades de agricultores especialmente en zonas áridas. Hace parte de lo que se empieza a denominar "nueva" conservación de suelos, porque el incremento de la MOS que está conectado con diversas funciones básicas del suelo, también significa mitigación de los GEI y del calentamiento global. Todo esto resulta, porque la MOS produce una "cascada de propiedades o funciones" que tienen relación con las propiedades del suelo, el efecto amortiguador, la capacidad de recuperación y la sostenibilidad. De ahí que sea tan importante el desarrollo de una agricultura de conservación en la que se toma muy en cuenta el contenido de carbono presente en la materia orgánica del suelo (Cotler, 2014; FA0, 2002).

La única forma de acumular carbono y evitar así la tasa de incremento de éste en la atmósfera es a través del secuestro de carbono, proceso que posibilita que el $\mathrm{CO}_{2}$ atmosférico se almacene en los distintos reservorios terrestres que se ubican en la biósfera, la hidrósfera, la pedosfera -en otras palabras los suelos del planeta- y la litosfera, en virtud de las uniones químicas del carbono con otras moléculas inorgánicas y orgánicas. Estos reservorios interactúan entre sí, mediante intercambios que realizan con la atmósfera (Miquelajauregui, 2013).

Lo más conocido respecto a la captura del carbono es la conversión del $\mathrm{CO}_{2}$ de la atmósfera en reserva orgánica de carbono o humus, como consecuencia de la vuelta al suelo de los residuos vegetales y la biomasa. Sin embargo, merced a otro proceso se forman carbonatos como los de calcio y magnesio, cuando se disuelve el $\mathrm{CO}_{2}$ en el aire del suelo y reacciona con el calcio y el magnesio presentes en los fertilizantes y la cal, así como de los biosólidos -estiércol entre otros- o como resultado de la deposición de polvo atmosférico. También hay captura de carbono en suelos bajo riego por efecto de la filtración de carbonatos con el movimiento del agua (Lal, 2003). Además de lo expresado, el secuestro de carbono constituye una opción que suma a las acciones para frenar la degradación del suelo y la calidad del mismo que influye positivamente en la salud de plantas, animales y seres humanos (Hontoria et al., 2004).

No obstante, debe advertirse que el carbono del suelo no se acumula para siempre, se va a liberar por descomposición de la MOS, que si bien por esta vía hace aporte de nutrientes para las plantas también libera $\mathrm{CO}_{2}$. Globalmente la respiración de los suelos es una fuente importante de $\mathrm{CO}_{2}$ atmosférico, como que contribuye al ciclo global del carbono con 100 billones de toneladas métricas anuales. Bajar este aporte que se origina en los suelos va a depender, como se expresaba previamente, de la gestión sostenible de la tierra que propugna por el almacenamiento del carbono en los suelos (Lessard et al., 2006). Este último objetivo se podrá alcanzar como lo señalan Orihuela y colaboradores (2012), con programas permanentes que evalúen las características de los suelos y el uso de la tierra 
en función de las tasas de acumulación de biomasa sobre el suelo, para tener elementos de juicio que hagan posible predecir la acumulación de carbono en el suelo en el ámbito de paisajes y regiones.

Quienes estudian el suelo como sumidero de carbono, hacen algunas distinciones que se deben tener en cuenta en el balance del carbono en este medio. Consideran que el punto en el que la cantidad de carbono en el suelo alcanza un equilibrio relativo para una situación particular de manejos se llama "capacidad de almacenamiento de COS", pero advierten que esta capacidad no necesariamente es el mayor nivel de carbono que un suelo pueda lograr. El COS llega a un máximo nivel de acumulación que se llama "punto de saturación", cuando la tasa de almacenamiento es igual a cero, no obstante, el ingreso de cantidades crecientes de de residuos orgánicos. Con estos argumentos, no recomiendan la utilización del nivel de carbono orgánico de suelos vírgenes como un referente del punto de saturación de carbono en el suelo (Martínez et al., 2008).

Para reiterar acerca de la relación que hay entre la producción de biomasa vegetal y la acumulación de carbono en la MOS, hay que referirse a las condiciones naturales y a las que resultan de la intervención de las primeras. Al respecto, el grupo de Caviglia (2016) distingue entre lo que ocurre en los ecosistemas naturales en donde las velocidades de formación y descomposición del carbono orgánico del suelo permanecen en un estado de equilibrio dinámico, muy controladas por la temperatura y las lluvias que pueden alterar tanto los aportes de residuos vegetales al suelo como las condiciones de descomposición según cada tipo de suelo. El opuesto según estos autores sucede en los agroecosistemas que, por efecto del manejo pueden modificar las entradas y salidas de residuos vegetales cambiando así la capacidad inicial de captura de $\mathrm{CO}_{2}$. El movimiento o desarreglo generado por la preparación del suelo favorece el meta- bolismo del carbono, porque lo expone a la acción de los microorganismos edáficos con la congruente emisión de $\mathrm{CO}_{2}$, que puede crecer si, además, se establecen especies vegetales que poco aportan residuos de fácil descomposición que incentivan la descomposición del COS a la cual sigue la salida del $\mathrm{CO}_{2}$ del medio edáfico (Caviglia et al., 2016).

El punto de partida para avanzar en la comprensión de los fenómenos que se vienen considerando, está ligado, sin duda, a contar con unas mediciones que cuantifiquen de la mejor manera los contenidos de carbono presentes en los en los diversos almacenes o depósitos terrestres, con una visión integral que tenga en cuenta amplios periodos de retorno y que los considere junto a las interrelaciones bióticas y abióticas, con miras a estimar, entre otros servicios ambientales, la potencialidad de captura de carbono que tienen los suelos (Álvarez et al., 2013).

Por consiguiente, para poder controlar y actuar sobre los procesos que determinan la presencia del carbono orgánico en el suelo, surge la necesidad e importancia de modelar el comportamiento del COS ligado a los manejos del suelo y al tiempo de duración de los mismos, utilizando la relación producción/descomposición (P/D) de residuos orgánicos. Si el COS tiene una condición estacionaria, la relación P/D es igual a uno; si el suelo se altera porque se hace agricultura convencional, el sistema funciona en forma transitoria y el P/D disminuye, porque hay más descomposición que producción de carbono. Dicho estado se mantiene hasta que se logra un nuevo nivel basal de COS, con una nueva condición estacionaria en la que el suelo no pierde más COS porque hay formas muy recalcitrantes que no permiten la mineralización. Si en este punto se establecen prácticas conservacionistas, hay una re-acumulación de COS -nueva situación estacionaria- con un cociente P/D superior a uno, situación que va hasta que se logre un nuevo nivel de equilibrio que puede ser menor, igual o mayor 
que la situación inicial del suelo antes de ser alterado (Martínez et al., 2008).

Posteriormente, surgieron modelos matemáticos para evaluar la dinámica y el balance del carbono en los ecosistemas a partir de información de campo. Dichos modelos se han orientado en dos direcciones. Unos, determinan sobre todo los pasos de transformación de la MOS y la energía, son los llamados modelos de proceso. Otros, cuantifican el movimiento del carbono por conteo directo de los agentes que descomponen la materia orgánica depositada (Orellana et al., 2012).

La investigación sobre secuestro de carbono que estudia la dinámica del COS, busca identificar los componentes de la MOS con diferente tasa de retorno mediante separación física. También se analizan los efectos del clima, clase de residuos y características del suelo como mineralogía, textura y estructura, entre otros, sobre el carbono orgánico. Como el uso del suelo influye en el contenido de MOS, la información sobre el particular, ha conducido a establecer modelos que predicen los cambios del carbono orgánico, como el Century y el DNDC en Estados Unidos y el RothC en Europa impulsado por la FAO (Hontoria et al., 2004).

Aceptando las limitaciones cuantitativas y temporales del suelo como sumidero de carbono, la opción de utilizar este recurso natural para almacenar carbono y mitigar el cambio climático es idónea, entre otras razones, porque: se puede emplear de manera inmediata, por cuanto no es preciso probar nuevas tecnologías; se conoce bien, por cuanto se trata de un proceso natural; no resulta costoso, aunque sí demanda un cambio en las prácticas autofinanciable a un mediano plazo. Más bien, el desafío está en lo social y en lo educativo, porque de lo que se trata es que los usuarios de la tierra y los decisores políticos valoren lo importante que es la gestión de la MOS por el potencial que tiene para contrarrestar la desertificación y contribuir a la mitigación del cambio climático. A estos efectos habría que sumar la ayuda para garantizar la seguridad alimentaria por la subida en los rendimientos agrícolas y por la mayor eficiencia de insumos agrícolas como los fertilizantes, derivada de unos adecuados contenidos de materia orgánica en el suelo. Por estas razones, desde hace algún tiempo la MOS es el atributo del suelo que se emplea con más frecuencia como indicador clave de su calidad y sostenibilidad agronómica, dado que tiene un amplio espectro de acción en lo físico, químico y biológico (Galantini y Suñer, 2008; Dimas y Gnacadja, 2009; Lal, 2009).

Para concluir este acápite y tomando en cuenta el conjunto, se necesita una visión integral de la naturaleza y de la vida, que siempre incluya al suelo por los roles que este desempeña en el planeta, al amparo de la ciencia y de la educación, para que, finalmente, esta concepción conduzca a un manejo respetuoso del suelo que se inserte en la cultura, con el acompañamiento de los grupos sociales y de quienes toman las decisiones políticas (Burbano, 2013).

\section{La iniciativa 4 por mil: consenso mundial para resolver un problema global}

A finales del 2015 tuvo lugar en París la vigésima primera sesión de la Conferencia de las Partes de la Convención Marco de Naciones Unidas sobre el Cambio Climático o COP21, que concluyó con un Acuerdo cuyo objetivo fundamental es "evitar que el incremento de la temperatura media global supere los $2^{\circ} \mathrm{C}$ respecto a los niveles preindustriales y busca, además, promover esfuerzos adicionales que hagan posible que el calentamiento global no supere los $1,5^{\circ} \mathrm{C}$. El Acuerdo pretende que para la segunda mitad del siglo XXI se logre "un equilibrio entre las emisiones y las absorciones de gases de efecto invernadero" (Ministerio de Agricultura y Pesca Alimentación y Medio Ambiente. Gobierno de España, 2015). 
Fue al interior de la COP21 que surgió el proyecto "4 por mil de suelos para la seguridad alimentaria y el clima", con el propósito de aumentar mundialmente la materia orgánica del suelo en 4 por 1000 o lo que es lo mismo en un 0,4\% al año, a fin de compensar las emisiones planetarias de gases de efecto invernadero derivadas de fuentes antropogénicas y detener así el incremento de la concentración de $\mathrm{CO}_{2}$ en la atmósfera (Minasny et al., 2017; Ministerio de Agricultura, Sector Agroalimentario y Bosques, Francia, 2015).

Para llevarla a la práctica, la iniciativa 4 por 1000 incluye dos grandes programas de actividades.

Un programa de acciones con diversos actores del Estado y de la sociedad civil, que mejore la gestión del carbono de los suelos, combata la pobreza y la inseguridad alimentaria, y que en paralelo contribuya a la adaptación al cambio climático porque mitiga las emisiones, mediante prácticas que restauren el suelo, incrementen sus reservas de carbono orgánico y protegen los suelos con grandes reservas de carbono, así como la biodiversidad. Incluye la capacitación y difusión de conocimientos sobre los aspectos de interés, la financiación de proyectos que restauren, mejoren y conserven las reservas de carbono en los suelos, junto con el suministro de productos agrarios amigables con los suelos (Ministerio de agricultura, Sector Agroalimentario y Bosques, Francia, 2015).

Un segundo programa internacional de investigación y cooperación científica que estudia los mecanismos y el potencial de almacenamiento de carbono en los suelos para regiones y sistemas identificados; que evalúa prácticas agrícolas idóneas y su impacto en la captura de $\mathrm{CO}_{2}$ y en los demás servicios de producción y regulación; que acompaña y promueva innovaciones con po- líticas pertinentes; que monitorea y evalúa los cambios en las reservas de carbono de los suelos en función del interés de los productores (Ministerio de Agricultura, Sector Agroalimentario y Bosques, Francia, 2015).

La iniciativa ha generado gran interés entre los investigadores y por eso ya hay planteamientos y análisis al respecto, tomando en cuenta la situación de varios países ubicados en diferentes continentes.

Al considerar 20 regiones del mundo el grupo de Minasny (2017) conceptúa que hay acciones para secuestrar carbono en el suelo que, merced a buenas prácticas hasta podrían superar el 4 por mil, tal el caso de suelos con bajos contenidos iniciales de carbono en un periodo estimado de 20 años luego del establecimiento de mejores prácticas de manejo, aunque se advierte que suelos que alcancen el equilibrio ya no podrían incrementar más el secuestro de carbono (Minasny et al., 2017).

El mismo grupo de investigación aconseja que en las circunstancias del cambio climático, el secuestro del carbono orgánico del suelo se deba establecer de inmediato y define un periodo de un decenio, en tanto surgen nuevas y eficaces tecnologías para el secuestro en asocio con bajas emisiones de carbono. Aunque, para avanzar en el 4 por mil se reclama una labor sincronizada entre científicos, agricultores, políticos y comerciantes. Estos investigadores también conceptúan que el 4 por mil es una aspiración ambiciosa, aunque celebran que, por primera vez, esta iniciativa asuma un objetivo global que aliente el buen manejo del suelo y que en paralelo ayude a mitigar el cambio climático (Minasny et al., 2017). 


\section{CONCLUSIONES}

El cambio climático global, guarda una clara relación con procesos de naturaleza antrópica que, a su vez, se entrelazan con los modelos de desarrollo que dominan en el mundo y de contera, en estas circunstancias, dicho cambio representa uno de los mayores problemas ambientales que la sociedad mundial deberá resolver para velar por la continuidad de la vida en el planeta.

Frente a este problema global, el suelo y su componente biorgánico pueden contribuir a mitigar el cambio climático, en la medida en que se racionalice el uso del suelo y se preserve el carbono orgánico del mismo, mediante prácticas de manejo idóneas que inclinen la balanza a favor de la fijación o secuestro de carbono, antes que a la salida del carbono del sistema suelo, con la consiguiente formación de gases de efecto invernadero.

Para contribuir a la mitigación del cambio climático y en la perspectiva de este documento, se requiere un estudio profundo y sincronizado de las formas y dinámica del carbono de los suelos de las regiones y del mundo, para contar con soportes científicos que apoyen decisiones sociales y políticas, en pro de la preservación global del carbono del suelo, de la sostenibilidad del suelo y de las funciones ambientales que este cumple en la red de la naturaleza, a fin de garantizar la seguridad alimentaria de una población que crece inexorablemente.

Conflicto de intereses: El autor declara que no hay conflicto de interés.

\section{REFERENCIAS BIBLIOGRÁFICAS}

Agencia Europea del Medio Ambiente. (2015). El suelo y el cambio climático. Recuperado de http://www. eea.europa.eu/es/senales/senales-2015/articulos/el-suelo-y-el-cambio-climatico
Altieri, M. \& Nicholls, C. (2013). Agroecología y resiliencia al cambio climático: principios y consideraciones metodológicas. Agroecología. 8(1): 7-20.

Álvarez, G., García, N. E., Krasilnikov, P. \& García, F. (2013). Almacenes de carbono en bosques montanos de niebla de la sierra norte de Oaxaca, México. Agrociencia. 47: 171-180.

Armas, C.M., Mora, J.L., Arbelo C.D. \& Rodríguez, A. (2013). Labile carbon pools and biological activity in volcanic soils of the Canary Islands. SJSS. Spanish Journal of Soil Science. 3(1): 7-27.

Blasco, M. \& Burbano, H. (2015). La vida en el suelo. Notas sobre su bioquímica y microbiología. Pasto: Impresos La Castellana. 365p.

Burbano, H. (2013). La sociedad depende del todo y las partes: naturaleza y suelo. Tendencias. 14(2): 9-22.

Caviglia, O.P., Wingeyer, A.B. \& Novelli, L. E. (2016). El rol de los suelos agrícolas frente al cambio climático. Serie de Extensión INTA Paraná. 78:27-32.

Cotler, H. (2014). Conservación de suelos y adaptación al cambio climático. México, Instituto Nacional de Ecología y Cambio Climático INECC. Recuperado de http://www.geologia.unam.mx:8080/igl/docs/difusion/simposios/2014/Suelos/1600\%20SuelosCotler-5Diciembre.pdf

Cotler, H., Martínez, M. \& Etchevers, J. D. (2016). Carbono orgánico en suelos agrícolas de México: investigación y políticas públicas. Terra Latinoamericana. 34: 125-138.

Dimas, S. \& Gnacadja, L. (2009). Prefacio. En: Cambio climático: ¿el suelo puede cambiar las cosas? Informe de la conferencia. Bruselas 12 de junio de 2008. Luxemburgo: Comisión Europea. 7-8 p.

Docampo, R. (2010). La importancia de la materia orgánica del suelo y su manejo en producción frutícola. Serie Actividades de Difusión № 687. Las Brujas: INIA -Estación Experimental "Wilson Ferreira Aldunate". 81 - 88p.

Equihua, M., Hernández, A., Pérez, O., Benítez, G. \& Ibáñez, S. (2016). Cambio global: el Antropoceno. CIENCIA ergo-sum. 23(1): 67-75. 
FA0 - Organización de las Naciones Unidas para la Alimentación. (2002). Captura de carbono en los suelos para un mejor manejo de la tierra. Basado en el trabajo de Michel Robert. Informes sobre recursos mundiales de suelos. FA0. 61p.

FAO - Organización de las Naciones Unidas para la Alimentación. (2015). El trabajo de la FAO sobre cambio climático. Conferencia de las Naciones Unidas sobre el cambio climático. Recuperado de http:// eird.org/ran-sendai-2016/presentaciones-eventoparalelo/documentos-de-interes/El-trabajo-de-laFAO-sobre-Cambios-Climatico-2015.pdf

Fuentes, M. H., Etchevers, J. D. \& Briones, O. (2012). El papel del programa mexicano del carbono en México en relación a los suelos. pp. 45-50. In: Paz, Fernando, Bazán, Maira, Saynes, Vinisa eds. Dinámica del carbono en el suelo 2012. Serie Avances Temáticos del Ciclo del Carbono y sus Interacciones. Texcoco, Estado de México, México: Programa Mexicano del Carbono en colaboración con la Sociedad Mexicana de Ciencias del Suelo. 208p.

Gabathuler, E., Liniger, H., Hauert, C. \& Giger, M. (2009). Beneficios de la gestión sostenible de la tierra. Germany: Wachs, Ted \& Thobault, Marlene, eds. Bern, WOCAT, CDE \& University of Berne. 16p.

Galantini, J.A. y Suñer, L. (2008). Las fracciones orgánicas del suelo: análisis en los suelos de la Argentina. AgrisScientia. 25(1): 41-55.

Gallardo, J. (2002). El agotamiento de la reserva orgánica del suelo y su relación con el secuestro de carbono atmosférico. Octavo Congreso Ecuatoriano de la Ciencia del Suelo. Recuperado de http://www. secsuelo.org/wp-content/uploads/2015/06/1Agotamiento-de-la-reserva-organica-Gallardo-J.pdf

Hontoria, C., Rodríguez, J. C. \& Saa, A. (2004). Contenido de carbono orgánico en el suelo y factores de control en la España peninsular. Edafología. 11(2): 149-157.

Kurniatun, H., Agus, F., Velarde, S. \& Van Noordwijk, M. (2011). Medición del carbono de los usos de la tierra. Capítulo 5. pp 5-30. In: White, Douglas y Minang, Peter, eds. Estimación de costos de oportunidad de REDD+. Manual de capacitación. Versión 1.4. Washington: Banco Mundial.
Labrador, J. (2012). Avances en el conocimiento de la dinámica de la materia orgánica dentro de un contexto agroecológico. Agroecología. 7: 91-108.

Lal, R. (2003). El cambio climático global y la dinámica del carbono en el suelo. IAI NewsLetter. 32: 28-30

Lal, R. (2009). La función de la materia orgánica del suelo en el ciclo global del carbono. Recuperado de http://ec.europa.eu/environment/archives/soil/ pdf/report_conference_es.pdf

Lessard, R., Gignac, L. D. \& Rochette, P. (2006). El ciclo del carbono: Midiendo el flujo del $\mathrm{CO}_{2}$ del suelo. Recuperado de https://greenteacher.com/article $\% 20$ files/elciclodelcarbono.pdf

Martínez, H., E., Fuentes E., J. P. \& Acevedo H., E. (2008). Carbono orgánico y propiedades del suelo. R.C. Suelo Nutr. Veg. 8(1): 68-96.

Minasny, B., Malone, B. P., McBratney, A. B., Angers, Dominique Arrouays, D. A., Chambers, A., Chaplot, V., Chen, Z. Chengg, K., Das, B. S., Field, D. J., Gimona, A., Hedley, C. B., Young Hong, S., Mandal, B., Marchant, B. P., Martin, M., McConkey, B. G., Mulder, V. L., O'Rourke, S., Richer-de-Forges, A. C., Odeh, I., Padarian, J., Paustian, K., Pan, G., Poggio, L., Savin, I., Stolbovoy, V., Stockmann, U., Sulaeman, Y., Tsui, Ch., Vågen, T. G., vanWesemael, B., Winowiecki, L.. (2017). Soil carbon 4 per mille. Geoderma. 292: 59-86.

Ministerio de Agricultura y Pesca Alimentación y Medio Ambiente, Gobierno de España. (2015). Resultados de la COP 21. P. 1. Recuperado de http://www.mapama.gob.es/es/cambio-climatico/temas/cumbrecambio-climatico-cop21/resultados-cop-21-paris/ default.aspx

Ministerio de Agricultura, Sector Agroalimentario y Bosques, Francia. (2015). La iniciativa 4 por 1000. Los suelos como base de la seguridad alimentaria y el clima. 7p. Recuperado de http://www.neiker.net/ wp-content/uploads/2015/11/4POR100.pdf.

Miquelajauregui, Y. (2013). Modelos de simulación de la dinámica del carbono. pp. 15-38. En J.A. Blanco Ed. Aplicaciones de modelos ecológicos a la gestión de recursos naturales. Barcelona: OmniaScience. 
Orellana, G., Sandoval, M. L., Linares, G., García, N. E. \& Tamariz, J. V. (2012). Descripción de la dinámica de carbono en suelos forestales mediante un modelo de reservorios. Avances en Ciencias e Ingeniería. 3(1): 123-135.

Orihuela, E., Mendoza, J., De Jong, B. \& Paz-Pellat, F. (2012). Carbono orgánico del suelo asociado a cronosecuencias de la reserva de la biosfera "Selva El Ocote”. pp. 87-92. In: Paz, Fernando, Bazán, Maira, Saynes, Vinisa eds. Dinámica del carbono en el suelo 2012. Serie Avances Temáticos del Ciclo del Carbono y sus Interacciones. Texcoco, Estado de México, México: Programa Mexicano del Carbono en colaboración con la Sociedad Mexicana de Ciencias del Suelo. 210p.

Pardos, J. A. (2010). Los ecosistemas forestales y el secuestro de carbono ante el calentamiento global. Madrid, Instituto Nacional de Investigación y Tecnología Agraria y Alimentaria (INIA). Madrid: Ministerio de Ciencia e Innovación. 253 p.

Pla, I. (2010). Ciencia del suelo y cambio climático, perspectivas para el futuro. XII Congreso Ecuatoriano de la Ciencia del Suelo. Santo Domingo, Ecuador. Recuperado de http://www.secsuelo.org/4159-xiicongreso-ecuatoriano-de-la-ciencia-del-suelo.html

PNUMA-ORPALC / FS-UNEP CENTRE. (2014). La agricultura andina frente al cambio climático. Documento del proyecto Microfinanzas para la adaptación basada en Ecosistemas (MEbA). Panamá: PNUMA-ORPALC / FS-UNEP CENTRE. 64p.

Rügnitz, M. T., Chacón, M. L. \& Porro, R. (2009). Guía para la determinación de carbono en pequeñas propiedades rurales. Primera edición. Lima, Perú: Centro Mundial Agroflorestal (ICRAF)/Consórcio Iniciativa Amazônica (IA). 79p.

Sandoval, M., Stolpe, N., Zagal, E., Mardones, M. \& Montano, J. (2003). El secuestro de carbono en la agricultura y su importancia con el calentamiento global. Theoria. 12: 65-71.

UNCCD - Convención de las Naciones Unidas de Lucha contra la Desertificación. (2015). Informe PolíticoCientífico № 01: 1-6. UNCCD.
Urquiaga, S., Jantalia, C. P., Luzio, W., Alves, B. \& Boddey, R. M. (2005). El horizonte del suelo. R.C. Suelo Nutr. Veg. 5(2): 46-60.

Vela, G., López, J. \& Rodríguez, M. de L. (2012). Niveles de carbono orgánico total en el Suelo de Conservación del Distrito Federal, centro de México. Investigaciones Geográficas. 77: 18-30

Verhulst, N., François, I. \& Govaerts, B. (2015). Agricultura de conservación y captura de carbono en el suelo: Entre el mito y la realidad del agricultor. México: CIMMYT. 16p.

Zambrano, A., Franquis, F. \& Infante, Á. (2004). Emisión y captura de carbono en los suelos en ecosistemas forestales. Rev. For. Lat. 35: 11-20. 